\title{
Adaptive Decomposition of Functions Into Pieces of Non-negative Instantaneous Frequencies
}

\author{
Tao Qian \\ Department of Mathematics, FST, University of Macau, Taipa, Macau, P.R.China, \\ fsttq@umac.mo \\ I.T.Ho \\ Department of Mathematics, FST, University of Macau, Taipa, Macau, P.R.China, \\ ma66530@umac.mo \\ I.T. Leong \\ Department of Mathematics, FST, University of Macau, Taipa, Macau, P.R.China, \\ ITLeong@umac.mo \\ Yanbo Wang \\ Department of Mathematics, FST, University of Macau, Taipa, Macau, P.R.China, \\ ya77405@umac.mo \\ Received (Day Month Year) \\ Revised (Day Month Year) \\ Communicated by (Yanbo Wang)
}

\begin{abstract}
We introduce the concept adaptive decomposition of signals into basic building blocks, of which each of non-negative analytic instantaneous frequency, called monocomponents. We propose certain methods based on $p$-starlike functions and Fourier expansions for such decomposition. We justify the terminology mono-component used in signal analysis.

Keywords: Hilbert transform, Hardy space, Fourier series, Fourier integral, analytic signal, instantaneous frequency, positivity of frequency, time-frequency analysis
\end{abstract}

AMS Subject Classification: 42A50, 32A30, 32A35, 46J15

\section{Introduction}

In signal analysis one seeks for representations of signals that provide at the same time the amplitude and phase (frequency) information. They are called amplitudephase representations, and are with the form

$$
f(t)=\rho(t) \cos \theta(t), \quad \rho(t) \geq 0 .
$$

Such representation, however, is not unique (see [3]). In order to have a determined amplitude-phase representation one introduces the so called analytic signal associ- 
ated with $f(t)$, defined by

$$
A(f) \triangleq f+i H f,
$$

where $H$ is Hilbert transformation on the line. Writing the associated analytic signal $A(f)$ into its amplitude-phase form, we have

$$
A(f)(t)=\rho(t) e^{i \theta(t)} .
$$

Thus

$$
f(t)=\rho(t) \cos \theta(t)
$$

with

$$
H f(t)=\rho(t) \sin \theta(t)
$$

or

$$
H(\rho(\cdot) \cos (\cdot))(t)=\rho(t) \sin \theta(t) .
$$

Use of analytic signals was traced back to Gabor ([5]). We call the above defined representation via the associated analytic signal the analytic amplitude-phase representation of $f$. One would further require that $\theta^{\prime}(t)$ exists a.e., and

$$
\theta^{\prime}(t) \geq 0, \quad \text { a.e. }
$$

The requirement is justified from its physics meaning. Indeed, frequency is a physics concept that represents the times of a certain vibration during the unit time interval, and hence it ought to be non-negative. If (1.3) holds, then we say that the instantaneous frequency exists ([2], [10]), and is $\theta^{\prime}(t)$. Only in this case, the instantaneous amplitude and instantaneous phase are considered to exist and are respectively defined to be $\rho(t)$ and $\theta(t)$.

The theory will be available in the parallel two contexts, that is the real line, $\mathbb{R}$, and the unit circle, $\partial \mathbb{D}$, where $\mathbb{D}$ denotes the unit disc. In the unit circle case the circular Hilbert transformation, denoted by $\tilde{H}$, will be in place of the Hilbert transformation $H$ on the line. For the definitions and basic properties of $H$ and $\tilde{H}$ we refer to [6] and [19]. It is known that a complex-valued function, $f$, in $L^{p}, p \in[1, \infty]$, is the boundary value of a function in the complex Hardy space $H^{p}$ if and only if its imaginary part is the Hilbert transform of its real part; and if and only if $H f=-i f$, modulo constants in the $L^{\infty}(\mathbb{R})$ case; and if and only if the spectrum of the function is contained in $[0, \infty)$. For $1<p<\infty$, the norm $\|A f\|_{p}$ is equivalent with the norm $\|f\|_{p}$. The function $A f$ is, in fact, the boundary value of the Cauchy integral of the boundary data $2 f$, while the Cauchy integral itself is one in the Hardy $H^{p}$ space. The above mentioned results for the range $1<p<\infty$ are quite standard and may be referred to [6] and [19]. For the cases $p=1$ and $p=\infty$ there is a treatment in [12] and [16]. Note that in the present paper we concentrate in the main idea. Therefore, the function spaces we deal with are restricted to the $L^{2}$ spaces, i.e. the spaces of signals of finite energy. 
It is a fact that the non-negativity requirement on analytic phase derivative (1.3) is not met by general signals. In fact, if $f$ is an outer function in $H^{\infty}(\mathbb{D})$ with analytic extension across the entire boundary $\partial \mathbb{D}$, then its phase derivative is of zero mean and therefore sometimes positive and sometimes negative ([14]). In relation to the negative phase derivative phenomenon there have been controversies to the concept instantaneous frequency among signal analysts. There is a mainstream, however, to believe that the concept instantaneous frequency is meaningless for general signals, but only meaningful for those who have non-negative analytic phase derivative, called mono-components (See the definition below). Those that are not mono-components, are called multi-components. In accordance with this one seeks for decompositions of multi-components into mono-components. Precisely, one is to decompose a given signal $f$ into a series of the form

$$
f(t)=\sum_{k=1}^{N} \rho_{k}(t) \cos \theta_{k}(t)+r_{N}(t),
$$

where $r_{N}$ is a small error term, and for each $k$, the following three requirements are met: (i) $\rho_{k} \geq 0$; (ii) $H\left(\rho_{k} \cos \theta_{k}\right)=\rho_{k} \sin \theta_{k}$; and (iii) The phase derivative $\theta_{k}^{\prime}$ may be properly defined and $\theta_{k}^{\prime} \geq 0$, a.e.

Remark 1.1. The above required conditions (i) and (ii) are automatically satisfied by the analytic amplitude $\rho_{k}$ and the analytic phase $\theta_{k}$, but the condition (iii) may not be, and turns to be critical. Since the phase derivative $\theta_{k}^{\prime}$ may not exist in the ordinary derivative sense, the condition (iii) is to be elaborated as follows (see also [14]). We take the signals $f(t)$ defined on the real line as example, those defined on the unit circle are similar. In the case $f+i H f$ is the non-tangential boundary limit of a function $F(z), z=t+i y, y>0$, in the Hardy $H^{2}\left(\mathbb{C}^{+}\right)$space. For any $y>0$, $F(t+i y)=\rho_{y}(t) e^{i \theta_{y}(t)}$, where $\theta_{y}(t)$ is well parameterized by $t$, and $\theta_{y}^{\prime}(t)$, in the ordinary derivative sense, is well defined. If the non-tangential limit

$$
\lim _{x+i y \rightarrow t} \theta_{y}^{\prime}(x)
$$

exists, then we say that $\theta^{\prime}(t)$ exists. If, moreover, the above defined $\theta^{\prime}(t)$ through non-tangential limit is non-negative a.e., then we say that the condition (iii) is met. It may be shown that if the analytic function $F(t+i y)$ has analytic continuation across an open interval $I$ on the line, then on $I$ the above defined derivative as a non-tangential limit of the same quantity but from inside of the domain coincides with the derivative $\theta^{\prime}(t)$ in the ordinary sense with the parametrization $t$ on the boundary.

The proof of Lemma 2.2 below will have to confront with this general definition of $\theta^{\prime}(t) \geq 0$. It can be easily shown that a function in the so called Hardy-Sobolev space has the above defined general phase derivative $\theta^{\prime}(t)$ (See the proof of Lemma $2.2)$. Whether it enjoys the non-negative property, however, is another issue.

Remark 1.2. We have used the terminology non-tangential limit. To clarify its meaning we make the following explanation. The usage of non-tangential is standard 
that means taking limit to a boundary point from inside a truncated cone whose axis is perpendicular to the tangent line of the boundary curve at the point ([6], [19]). Below, a limit, as a function, exists amounts to say that limits exist a.e. When we say that a function is non-zero or non-negative, they mean that the function values are non-zero a.e. or non-negative a.e.

Definition 1.1. A function $\rho(t) \cos \theta(t)$ satisfying the above listed three conditions (with Remark 1.1 on condition (iii)) is called a real mono-component or a mono-component on the line. A complex-valued signal $\rho(t) e^{i \theta(t)}$ is a complex-monocomponent if and only if its real part $\rho(t) \cos \theta(t)$ is a real mono-component. One similarly defines real mono-component and complex mono-component on the unit circle through replacing $H$ by $\tilde{H}$.

In the complex-valued case the required condition (ii) in relation to Hilbert transformation is equivalent to the eigen-value condition $H\left(\rho(\cdot) e^{i \theta(\cdot)}\right)(t)=-i \rho(t) e^{i \theta(t)}$. An analytic signal $A f=\rho e^{i \theta}$ that does not satisfy the three conditions is called a complex multi-component. The associated real-valued signal $f$ is called a real multicomponent. Note that if $f$ is a multi-component, then its analytic amplitude-phase representation satisfies the conditions (i) and (ii), but not (iii), and its instantaneous frequency does not exist.

The above definition of mono-components first appeared in [13]. In signal analysis use of the terminology mono-component and multi-component has had a long history. In [3], and the related literature, complex-valued signals of the form $\rho(t) e^{i \theta(t)}$, where $\rho(t)$ is tacitly assumed to be a Gaussian density and $\theta$ a polynomial, are called "mono-components," and are studied in great detail. In order to avoid ambiguity in relation to amplitude-phase representation of real-valued signals some literature does not concern the concept mono-component for real-valued signals. The reference [3], for instance, presents a number of paradox arguments in relation to analytic phase derivative. In Boashash [2] and the related literature mono-components for real-valued signals are defined through analytic signals in a similar way. The positivity of the phase derivative is often assumed when deducing their results. They further require invertibility of the phase derivative. Under these assumptions they show that the inverse function of the phase derivative coincides in the asymptotic sense with the function law of group delay. We, however, would insist on the positivity of the phase derivative. The article [2] also gives a comprehensive historical review on development of the related concepts.

The Fourier series expansion of $f \in L^{2}(\partial \mathbb{D})$ is an example of the expansion (1.4). We note that due to the relations

$a_{k} \cos k t=\left|a_{k}\right| \cos \left(k t+\frac{1-\operatorname{sgn}\left(a_{k}\right)}{2} \pi\right), \quad b_{k} \sin k t=\left|b_{k}\right| \cos \left(k t-\frac{\pi}{2}+\frac{1-\operatorname{sgn}\left(b_{k}\right)}{2} \pi\right)$,

any function of the forms $a \cos k t$ and $b \sin k t$ is real mono-component. A Fourier series, however, usually converges slowly. The reason is that they use all the basis functions in the trigonometric system, some of which are not intrinsic components 
of the signal. An adaptive decomposition (1.4) may achieve fast convergence in a certain sense. For instance, we may require, for a given $\epsilon>0$, to find the minimal integer $N$ and, at the same time, $N$ specific mono-components, whose sum approximates $f$ within an error less than $\epsilon$ in the metric adopted in the space. Or, alternatively, we can first fix $N$ and seek for $N$ specific mono-components whose sum provides the best approximation to $f$ in the given metric space. Note that adaptive decomposition into mono-components is not of uniqueness, but application dependent (also see Remark 2.1). The composing mono-components may not be orthogonal to each other either. As a matter of fact, the totality of mono-components consists of many more basic functions than what is necessary to form a base, and, in particular, it contains the trigonometric system. This makes adaptive decomposition possible. In the present paper we provide theoretical examples for such decomposition, and show what properties can we expect from such decompositions. With combined effort of different researchers there have found a large pool of monocomponents in both the real line and the unit circle contexts, including boundary values of (i) Möbius transforms (Fourier atoms) and finite Blaschke products, in which the trigonometric functions are particular cases ([10],[11],[12],[15]); (ii) inner functions, consisting of infinite Blaschke products and singular inner functions [14]; (iii) $p$-slarlike functions $([13],[17],[22])$; and (iv) weighted forms of the Fourier atoms and their finite products based on various Bedrosian Theorems ([16],[20],[21]).

The analytic amplitude-phase representation of a mono-component gives a time varying (analytic) instantaneous frequency and a instantaneous amplitude. In signal analysis such representation are desirable. Indeed, amplitude and frequency are among the most striking physical properties of a signal. The above decomposition will have the best localization property. We note that each complex monocomponent may be said to be physically realizable as it is the boundary value of an analytic function.

To the authors, the motivation of the study is the algorithm called Empirical Mode Decomposition (EMD) , [8]. The EMD algorithm and its variants may be described as follows. Let $f$ be a given data. Denote by $E_{u} f$ the upper envelop formed through connecting the consecutive local maximum points of $f$ using splines of, say, order 3 ; and $E_{l} f$ the lower envelop formed through connecting the consecutive local minimum points of $f$ using the same kind of splines. Denote by $M f$ and $D f$, respectively, the operators

$$
M f=\frac{1}{2}\left(E_{u} f+E_{l} f\right), \quad D f=f-M f .
$$

Repeating this iteration up to the $n_{1}$ step at which we first have $D^{n_{1}} f \approx D^{n_{1}-1} f$ in accordance with a previously set error threshold. This implies that $M D^{n_{1}-1} f=$ $D^{n_{1}-1} f-D^{n_{1}} f \approx 0$. Set $f_{1}=D^{n_{1}-1} f$. To $f-f_{1}$ repeating the iteration until we arrive the first $n_{2}$ at which $D^{n_{2}}\left(f-f_{1}\right) \approx D^{n_{2}-1}\left(f-f_{1}\right)$. We set $f_{2}=D^{n_{2}-1}(f-$ $f_{1}$ ), and to $f-f_{1}-f_{2}$ repeat again the same procedure, and so on. With every subtraction in applying the operator $D$ we eliminate lower frequency components 
and thus finally obtain the component of the highest frequency. Proceeding like this we consecutively obtain $f_{1}, f_{2}, \ldots, f_{m}$, and thus arrive $f=f_{1}+\cdots+f_{m}+r_{m}$. The iteration process stops running owing to the previously set threshold. The obtained $f_{k}, k=1, \ldots, m$ are called Intrinsic Mode Functions (IMFs). The original signal is decomposed into a sum of IMFs together with an error term. The IMF functions $f_{k}$ satisfy $M f_{k} \approx 0$, and between each pair of adjacent local maximum and local minimum there is a zero crossing. The mentioned properties do not define the IMF functions but only describe those functions. To judge whether a function is an IMF associated with an EDM algorithm, the only method is to see whether it is produced by this particular EMD algorithm. Or, whether an EMD algorithm cannot further decompose it. The EMD-IMF decomposition has found ample engineering applications. However, IMFs do not possess the analytic properties of the cosine and sine functions. The article [18] works out examples showing that there exist IMFs whose analytic phase derivatives take opposite signs in adjacent open intervals. This shows that IMFs are not mono-components. The literature [17] provides further comparisons between mono-components and IMFs. Indeed, it is the algorithm itself that prevents IMFs from having good analytic properties.

There may be two basic approaches for the adaptive decomposition task (1.4). First, one can seek for a large pool of mono-components, and then try to compose a given signal by selecting the mono-components in the pool most suitable for the signal. Along this line the study has mainly been to find various types of mono-components. While this could be said quite successful, the progress for the composing part is very little. In the second approach, one would try to design an algorithm that automatically yields the composing mono-components. In this latter fold an algorithm like EMD would be ideal. As mentioned, the problem with EMD is that it does not produce mono-components. The study presented in the paper is a combination of the mentioned two approaches. It uses some mono-components in our pool, but it does not involve an effective algorithm like EMD. The study shows that an adaptive decomposition is theoretically possible. Precisely, for any $f$ in the underlying space and a given $\epsilon>0$, one can identify the smallest integer $N$ and correspondingly $N$ pairs of mono-components such that the sum of the $N$ pairs of mono-components approximates $f$ with an error less than $\epsilon$. Surprisingly, the smallest integer number $N$ is, as a matter of fact, equal to 1 , modulo the constant mono-components.

Our main results are stated as follows.

Theorem 1.1. Let $f \in L^{2}(\partial D)$ be real-valued, and $\epsilon_{n}, n=1,2, \ldots$, be a sequence of positive numbers strictly decreasing to zero. Then there exists a sequence of real mono-components, $T_{0}=c_{0}$, a constant, and $T_{1}\left(e^{i t}\right), \tilde{T}_{1}\left(e^{i t}\right), \ldots, T_{n}\left(e^{i t}\right), \tilde{T}_{n}\left(e^{i t}\right), \ldots$, such that

$$
\left\|f-\left[T_{0}+\sum_{k=1}^{n}\left(T_{k}+\tilde{T}_{k}\right)\right]\right\|_{2} \leq \epsilon_{n}, \quad n=1,2, \ldots
$$


Corollary 1.1. Let $f \in L^{2}(\partial D)$ be real-valued. For any $\epsilon>0$, there exist three real mono-components $T_{0}=c_{0}, T_{1}\left(e^{i t}\right)$ and $\tilde{T}_{1}\left(e^{i t}\right)$ such that

$$
\left\|f-\left(T_{0}+T_{1}+\tilde{T}_{1}\right)\right\|_{2} \leq \epsilon .
$$

Corollary 1.2. Let $f \in L^{2}(\partial D)$ be real-valued. If the Fourier series of $f$ satisfies the condition

$$
\sum_{k=2} k\left|c_{k}\right|<\infty
$$

then $f$ can be expressed as a sum of three real mono-components.

Denote by $\kappa$ the Cayley transformation that maps the upper-half complex space $\mathbb{C}^{+}$conformally onto the unit disc $\mathbb{D}$, and extends to a smooth and one to one mapping from $\mathbb{R}$ to $\partial \mathbb{D}$ :

$$
\kappa: \mathbb{C}^{+} \rightarrow \mathbb{D}, \quad w=\kappa(z)=\frac{i-z}{i+z}, \quad \kappa(i)=0, \quad \kappa(\infty)=-1,
$$

and

$$
e^{i t}=\kappa(s)=\frac{i-s}{i+s}, \quad t=2 \arctan s, \quad \frac{d t}{d s}=\frac{2}{1+s^{2}} .
$$

We will use the correspondence

$$
F(s)=f(\kappa(s))=f\left(e^{i t}\right),
$$

and $f\left(e^{i t}\right)$ is in $L^{2}(\partial \mathbb{D})$ if and only if $F(s)$ is in $L^{2}\left(\mathbb{R}, \frac{d s}{1+s^{2}}\right)$ with equal norms in the respective spaces up to a factor $\sqrt{2}$.

For functions defined on the whole real line we have

Theorem 1.2. Let $F \in L^{2}\left(\mathbb{R}, \frac{d s}{1+s^{2}}\right)$ be real-valued. Under Caylay transformation it corresponds to the function $f \in L^{2}(\mathbb{D})$. For a sequence $\epsilon_{n}, n=1,2, \ldots$, of positive numbers strictly decreasing to zero, let $T_{k}, \tilde{T}_{k}$ be the functions associated with $f$ defined in Theorem 1.4 and $S_{0}=T_{0}, S_{n}(s)=T_{n}(2 \arctan s), \tilde{S}_{n}(s)=$ $\tilde{T}_{n}(2 \arctan s), n=1,2, \ldots$ Then $S_{0}, S_{n}$ and $\tilde{S}_{n}, \cdots$ etc. are real mono-components in $L^{2}\left(\mathbb{R}, \frac{d s}{1+s^{2}}\right)$, and

$$
\left\|F-\left[S_{0}+\sum_{k=1}^{n}\left(S_{k}+\tilde{S}_{k}\right)\right]\right\|_{2} \leq \epsilon_{n}
$$

Correspondingly, we have

Corollary 1.3. Let $F \in L^{2}\left(\mathbb{R}, \frac{d s}{1+s^{2}}\right)$ be real-valued. For any $\epsilon>0$, there exist three real mono-components $S_{0}, S_{1}, \tilde{S}_{1}$ such that

$$
\left\|F-\left(S_{0}+S_{1}+\tilde{S}_{1}\right)\right\|_{2} \leq \epsilon,
$$

where $S_{1}$ and $\tilde{S}_{1}$ are in $L^{2}\left(\mathbb{R}, \frac{d s}{1+s^{2}}\right)$.

We offer a direct proof, by using Fourier inversion formula, of a set of results similar to Theorem 1.2 and its corollaries. 
Theorem 1.3. Let $F \in L^{2}(\mathbb{R})$ be real-valued, and $\epsilon_{n}, n=1,2, \ldots$, be a sequence of positive numbers strictly decreasing to zero. Then there exists a sequence of complex mono-components $G_{1}, \tilde{G}_{1}, \ldots, G_{n}, \tilde{G}_{n}, \ldots$, in $L^{2}\left(\mathbb{R}, \frac{d s}{1+s^{2}}\right)$, such that

$$
\left\|F-\operatorname{Re}\left[e^{-i(\cdot)} \sum_{k=1}^{n}\left(G_{k}+\tilde{G}_{k}\right)\right]\right\|_{2} \leq \epsilon_{n} .
$$

There are correspondingly the following corollaries.

Corollary 1.4. Let $F \in L^{2}(\mathbb{R})$ be real-valued. For any $\epsilon>0$, there exist two complex mono-components $G_{1}$ and $\tilde{G}_{1}$ in $L^{2}\left(\mathbb{R}, \frac{d s}{1+s^{2}}\right)$ such that

$$
\left\|F-\operatorname{Re}\left[e^{-i(\cdot)}\left(G_{1}+\tilde{G}_{1}\right)\right]\right\| \leq \epsilon .
$$

The following corollary is based on a set of assumptions inducing the SobolevHardy spaces.

Corollary 1.5. Let $F \in L^{2}(\mathbb{R})$ be real-valued with

$$
\int_{|\xi|>1} \xi^{2}|\hat{F}(\xi)|^{2} d \xi<\infty, \quad \int_{|\xi|>1} \xi|\hat{F}(\xi)| d \xi<\infty
$$

then there exist two complex mono-components $G_{1}, \tilde{G}_{1}$ in $L^{2}\left(\mathbb{R}, \frac{d s}{1+s^{2}}\right)$ such that

$$
F(s)=\operatorname{Re}\left[e^{-i s}\left(G_{1}(s)+\tilde{G}_{1}(s)\right)\right] .
$$

\section{Proofs of the Results}

For an analytic function $f: \mathbb{D} \rightarrow \mathbb{C}$, writing $f\left(r e^{i t}\right)=\rho_{r}(t) e^{i \theta_{r}(t)}, r<1$, and taking derivative to both sides of the equality with respect to $t$, we obtain that

$$
\frac{d}{d t} \theta_{r}(t)=\operatorname{Re}\left[z \frac{f^{\prime}(z)}{f(z)}\right] .
$$

This result may not be available for general boundary points on $|z|=1$, but available for those across which the function has an analytic continuation. On the boundary the substitution of the phase derivative is the angular limit or non-tangential limit of the phase derivative from inside the unit disc ([14]). Recall that a univalent analytic function $f$ in $\mathbb{D}$ is said to be starlike, or 1-starlike, about zero, if $f(0)=0$, and

$$
\operatorname{Re}\left[\frac{z f^{\prime}(z)}{f(z)}\right] \geq 0 \quad \text { for } 0 \neq z \in \mathbb{D} \text {. }
$$

Intuitively, a univalent analytic function is starlike if and only if the phase function $\theta_{r}(t)$ is increasing in $t$ for any $r \in(0,1)$, and $e^{i \theta_{r}(t)}$ traverses a full cycle when $e^{i t}$ does so. Generalizations of this concept include $p$-starlike functions (about zero). We recall the definition of $p$-valent starlike function as follows (see [7]).

Definition 2.1. The function $f(z)$, analytic in $\mathbb{D}$, is said to be in the class $S(p, m)$, or $p$-starlike about zero, where $p$ and $m$ are positive integers with $p \geq m$, if and only 
if

(i) there exists a positive $\rho<1$ such that

$$
\operatorname{Re}\left[\frac{z f^{\prime}(z)}{f(z)}\right] \geq 0, \quad \rho<|z|<1
$$

(ii)

$$
f(z)=z^{m}+a_{m+1} z^{m+1}+\cdots, \quad|z|<1 ; \text { and },
$$

(iii)

$$
\int_{0}^{2 \pi} \operatorname{Re}\left[\frac{z f^{\prime}(z)}{f(z)}\right] d t=2 \pi p, \quad z=r e^{i t}, \rho<r<1
$$

(i.e., $f(z)$ is $p$-valent in $|z|<1$ ).

In other words, $f(z)$ belongs to $S(p, m)$ if and only if $f(z)$ is $p$-valent starlike with respect to the origin and has an $m$-th order zero at $z=0$. There exists the following factorization of the class $S(p, m)$ :

$$
S(p, m)=[S(1,1)]^{m-1} S(p-m+1,1), \quad 1<m<p
$$

(see [7]).

We will use the following technical lemma ([9]).

Lemma 2.1. Let $f$ be analytic in $\mathbb{D}$ with the Taylor expansion

$$
f(z)=z+\sum_{k=2}^{\infty} a_{k} z^{k} .
$$

If

$$
\sum_{k=2}^{\infty} k\left|a_{k}\right|<1
$$

then $f(z)$ is starlike in $\mathbb{D}$.

For comparison with the proof of its real-line analogue, Lemma 2.2 below, we cite the simple proof of Lemma 2.1 ([9]).

Proof The inequality (2.3) implies that the winding number of $f\left(r e^{i t}\right)$ is 1 for all $0<r<1$ and therefore $f$ is univalent. For $|z|<1$, we have

$$
\begin{aligned}
\left|f(z)-z f^{\prime}(z)\right| & \leq \sum_{k=2}^{\infty}(k-1)\left|a_{k} z^{k}\right| \\
& <|z|-\sum_{k=2}^{\infty}\left|a_{k} z^{k}\right| \\
& \leq|f(z)| .
\end{aligned}
$$

Dividing the both ends of the above chain of inequalities by $|f(z)|$, we obtain

$$
\left|\frac{z f^{\prime}(z)}{f(z)}-1\right|<1, \quad z \neq 0 .
$$


Therefore,

$$
\operatorname{Re}\left[\frac{z f^{\prime}(z)}{f(z)}\right]>0, \quad z \neq 0 .
$$

Hence the function $f$ is starlike. The proof is complete.

Proof of Theorem 1.1 Let integers $N_{1}<\ldots<N_{n}<N_{n+1} \ldots$ be chosen so that $N_{1}>1$ such that

$$
\left\|f-\left(c_{0}+\sum_{1 \leq|k| \leq N_{n}} c_{k} e^{i k(\cdot)}\right)\right\|_{2} \leq \epsilon_{n}, \quad n=1,2, \ldots,
$$

where

$$
c_{k}=\frac{1}{2 \pi} \int_{0}^{2 \pi} f\left(e^{i t}\right) e^{-i k t} d t, \quad k \in \mathbb{Z} .
$$

The fact that $f$ is real implies that $c_{0}$ is real, and $c_{n}$ and $c_{-n}$ are complex conjugate to each other. We need to show that for every $n \geq 0$, with $N_{0}=0$, the partial sum

$$
\sum_{N_{n}+1 \leq|k| \leq N_{n+1}} c_{k} e^{i k t}
$$

that is real, may be expressed into a sum of two real mono-components. Since the $k>0$ part and the $k<0$ part of the partial sum are complex conjugate to each other, it will suffice to show that

$$
\sum_{N_{n}+1 \leq k \leq N_{n+1}} c_{k} e^{i k t}
$$

may be written as a sum of two complex mono-components. Their real parts will be correspondingly defined as $\frac{1}{2} T_{k}$ and $\frac{1}{2} \tilde{T}_{k}$, respectively, as stated in the theorem with the required properties. We will prove this by showing that the polynomial (2.4) is the sum of some two $p$-starlike functions. In fact, for any $n \geq 0$, for a large constant $A>0$, we have the decomposition

$$
\begin{aligned}
\sum_{N_{n}+1 \leq k \leq N_{n+1}} c_{k} z^{k}=z^{N_{n}} & \left.\left.\left(A+c_{N_{n}+1}\right) z+\frac{1}{2} \sum_{N_{n}+1<k \leq N_{n+1}} c_{k} z^{k-N_{n}}\right)\right) \\
& +z^{N_{n}}\left(-A z+\frac{1}{2} \sum_{N_{n}+1<k \leq N_{n+1}} c_{k} z^{k-N_{n}}\right) .
\end{aligned}
$$

Choose $A$ so that

$$
\frac{1}{2\left(A-\left|c_{N_{n}+1}\right|\right)} \sum_{N_{n}+1<k \leq N_{n+1}}\left(k-N_{n}\right)\left|c_{k}\right|<1 .
$$

Invoking Lemma 2.1, the above is the sum of some two starlike functions. The proof is complete.

Taking $\epsilon_{1}=\epsilon$, we obtain Corollary 1.1. To prove Corollary 1.2, we only need to point out that under the assumed condition we can, in the proof of the theorem, 
let the summation upper limit to be $\infty$. To prove Theorem 1.2 and its corollary we only need to notice that the mapping between $F(s)$ and $f\left(e^{i t}\right)$ under Cayley transformation is an isometric isomorphism with respect to the two respective $L^{2}$ spaces.

Remark 2.1. The decompositions in the above proved theorems and corollaries do not possess uniqueness. First, the choice of the increasing integers sequence $\left\{N_{n}\right\}$ is not unique. Once the sequence $\left\{N_{n}\right\}$ is chosen, the technical treatment in the proof of Theorem 1.4 for decomposing

$$
\sum_{N_{n}+1 \leq k \leq N_{n+1}} c_{k} e^{i k t}
$$

can have many variants. For instance, one can, instead, write, for a large constant $A>0$ and $0 \leq m \leq N_{n}-1, n>0$,

$$
\begin{aligned}
\sum_{N_{n}+1 \leq k \leq N_{n+1}} c_{k} z^{k}= & \frac{1}{2} z^{N_{n}-1-m}\left[\left(A z+\sum_{N_{n}+1 \leq k \leq N_{n+1}} c_{k} z^{k-N_{n}+m+1}\right)+\right. \\
& \left.\left(-A z+\sum_{N_{n}+1 \leq k \leq N_{n+1}} c_{k} z^{k-N_{n}+m+1}\right)\right]
\end{aligned}
$$

and choose

$$
A=A_{n} \geq \sum_{N_{n}+1 \leq k \leq N_{n+1}}\left(k-N_{n}+m+1\right)\left|c_{k}\right| .
$$

In the case, the obtained mono-components $T_{n}$ and $\tilde{T}_{n}$ are in $S\left(p, N_{n}-m\right), p \geq$ $N_{n}-m$. Furthermore, the technique of our decomposition, so far, is based on Lemma 2.1. This is, however, among many other sufficient conditions of starlike functions (See [13] or [17] for a summary, and also [22]). Being lack of uniqueness, however is anticipated. It is because of the non-uniqueness that makes the adaptive decompositions for various purposes possible.

Now we turn to prove Theorem 1.3. Let $F(z)$ be analytic in the upper-half complex plane. Writing $F(s+i y)=\rho_{y}(s) e^{i \phi_{y}(s)}, y>0$, and taking derivative to both sides of the equality with respect to $s$, we obtain

$$
\phi_{y}^{\prime}(s)=\operatorname{Im}\left[\frac{F^{\prime}(z)}{F(z)}\right], \quad \text { when } F(z) \neq 0 .
$$

By Definition 1.1 and Remark 1.1, when a function $F(s)$, originally defined on the line, may be extended to become a function in the Hardy space of the upperhalf plane, and the non-tangential boundary limit of the phase derivative given in (2.5) exists on $\mathbb{R}$, then the limit is defined to be the phase derivative on the line. Furthermore if the limit is non-negative, then the function $F(s)$ is a complex mono-component. 
We will first prove a lemma analogous to Lemma 2.1. The Fourier transformation adopted in this paper is defined through

$$
\hat{F}(\xi)=\int_{-\infty}^{\infty} e^{-i s \xi} F(s) d s .
$$

Under certain conditions we have the Fourier inversion formula

$$
F(s)=\frac{1}{2 \pi} \int_{-\infty}^{\infty} e^{i s \xi} \hat{F}(\xi) d \xi .
$$

Lemma 2.2. Let $F \in L^{2}(\mathbb{R})$ be real-valued and the support of its Fourier transform, $\hat{F}$, is contained in $[1, \infty)$. If

$$
\int_{1}^{\infty} \xi^{2}|\hat{F}(\xi)|^{2} d \xi<\infty
$$

and

$$
\int_{1}^{\infty} \xi|\hat{F}(\xi)| d \xi<1
$$

then

$$
G(s)=\frac{1}{2 \pi}\left(e^{i s}+\int_{1}^{\infty} e^{i \xi s} \hat{F}(\xi) d \xi\right)=\frac{1}{2 \pi} e^{i s}+F(s)
$$

is a complex mono-component.

Remark 2.2. When $\operatorname{supp} \hat{F} \subset[a, b]$, where $-\infty<a<b<\infty$, we have supp $\hat{F}_{1} \subset$ $[1, b-a+1]$, where $F_{1}(s)=e^{-i(a-1) s} F(s)$. If $A$ is large enough, then for $\frac{1}{A} F_{1}$ the conditions (2.6) and (2.7) are automatically satisfied. It is this case that is used in the following proof of Theorem 1.3. Only the proof of Corollary 1.2 uses the Lemma 2.2 to its greatest potential with the assumptions (2.6) and (2.7) necessary for the infinite interval $[1, \infty)$.

\section{Proof of Lemma 2.2}

$$
\begin{aligned}
\left|G^{\prime}(z)-i G(z)\right| & \leq \frac{1}{2 \pi} \int_{1}^{\infty}\left|e^{i \xi z}(\xi-1) \hat{F}(\xi)\right| d \xi \\
& <\frac{1}{2 \pi}\left(\left|e^{i z}\right|-\int_{1}^{\infty}\left|e^{i \xi z} \hat{F}(\xi)\right| d \xi\right) \\
& \leq|G(z)| .
\end{aligned}
$$

Therefore,

$$
\left|\frac{G^{\prime}(z)}{G(z)}-i\right|<1, \quad z \in \mathbb{C}^{+}, G(z) \neq 0
$$

This shows that

$$
\operatorname{Im}\left[\frac{G^{\prime}(z)}{G(z)}\right]>0, \quad z \in C^{+}, G(z) \neq 0 .
$$

Now we show that the left-hand-side of (2.9) has a non-tangential boundary limit. In fact, supp $\hat{F} \subset[1, \infty]$ implies that $F$ is in $H^{2}\left(\mathbb{C}^{+}\right)$and hence has a non-tangential 
boundary limit. Moreover, the boundary limit of $F$ is non-zero (p 65, [6]). This will imply that $G$ itself has a non-tangential boundary limit that is non-zero. In fact, if this were not true, then $F(s)=-i e^{i s}$ would hold for $s$ in a set of positive Lebesgue measure. Note that $\hat{F}$ is integrable implies that $F$ belongs to $H^{\infty}\left(\mathbb{C}^{+}\right)$. The function $e^{i z}$ also belongs to $H^{\infty}\left(\mathbb{C}^{+}\right)$. Since they coincided on a set of positive Lebesgue measure on the boundary, the two $H^{\infty}$ functions then would have to be identical (p 65, [6]). On the other hand $F(z)$ is in $H^{2}\left(\mathbb{C}^{+}\right)$, but $e^{i z}$ is not in $H^{2}\left(\mathbb{C}^{+}\right)$. This contradiction shows that the boundary limit of $G$ is non-zero. Next we show that $G^{\prime}(z)$ has a non-tangential boundary limit. The condition (2.6) implies that $F^{\prime}(z) \in H^{2}\left(\mathbb{C}^{+}\right)$(That is, $F$ is in the Sobolev-Hardy space). Therefore, $F^{\prime}(z)$ has a non-tangential boundary limit., and so does $G^{\prime}(z)$. These conclude that the nontangential boundary limit of the left-hand-side of (2.9) exists and is non-negative. To show that $G(s)$ is a mono-component we finally have to show $H G=-i G$. This eigenvalue relation, in fact, holds for both $e^{i s}$ and $F(s)$, and thus holds for their sum. The proof of the lemma is complete.

Proof of Theorem 1.3 Since $F \in L^{2}(\mathbb{R})$, we have the Hardy space decomposition

$$
F=F^{+}+F^{-},
$$

where

$$
F^{+}(z)=\frac{1}{2 \pi} \int_{0}^{\infty} e^{i \xi z} \hat{F}(\xi) d \xi, \quad z \in \mathbb{C}^{+}
$$

and

$$
F^{-}(z)=\frac{1}{2 \pi} \int_{-\infty}^{0} e^{i \xi z} \hat{F}(\xi) d \xi, \quad z \in \mathbb{C}^{-} .
$$

Since $F$ is real-valued on $\mathbb{R}$, we have

$$
\overline{\hat{F}}(\xi)=\hat{F}(-\xi)
$$

Now we consider the $F^{+}$part of $F$, whose spectra are restricted in the right-half of the real axis. Setting $F_{1}(s)=e^{i s} F^{+}(s)$, we introduce a shift of the spectra:

$$
F^{+}(z)=\frac{e^{-i z}}{2 \pi} \int_{1}^{\infty} e^{i \xi z} \hat{F}_{1}(\xi) d \xi .
$$

For the given sequence $\epsilon_{n}$ strictly decreasing to zero, we can find a strictly increasing integers sequence $1=N_{0}<N_{1}<\cdots$ such that

$$
\left\|F^{+}-\frac{e^{-i(\cdot)}}{2 \pi} \int_{1}^{N_{n}} e^{i \xi(\cdot)} \hat{F}_{1}(\xi) d \xi\right\|_{2} \leq \frac{\epsilon_{n}}{2}, \quad n=1,2 \ldots
$$

To prove the theorem it suffices to show that for each $n=0,1,2, \ldots$, there exist two complex mono-components $G_{n}(z)$ and $\tilde{G}_{n}(z)$ such that

$$
\frac{1}{2 \pi} \int_{N_{n}}^{N_{n+1}} e^{i \xi z} \hat{F}_{1}(\xi) d \xi=\frac{G_{n}(z)}{2}+\frac{\tilde{G}_{n}(z)}{2} .
$$


Set, for a positive number $A_{n}, n=1,2, \ldots$, to be determined later,

$$
\begin{aligned}
& G_{n}(z)=\frac{A_{n} e^{i\left(N_{n}-1\right) z}}{2 \pi}\left(e^{i z}+\frac{1}{A_{n}} \int_{1}^{N_{n+1}-N_{n}+1} e^{i \xi z} \hat{F}_{1}\left(N_{n}-1+\xi\right) d \xi\right), \\
& \tilde{G}_{n}(z)=\frac{A_{n} e^{i\left(N_{n}-1\right) z}}{2 \pi}\left(-e^{i z}+\frac{1}{A_{n}} \int_{1}^{N_{n+1}-N_{n}+1} e^{i \xi z} \hat{F}_{1}\left(N_{n}-1+\xi\right) d \xi\right) .
\end{aligned}
$$

Choosing

$$
A_{n} \geq \int_{1}^{N_{n+1}-N_{n}+1} \xi\left|\hat{F}_{1}\left(N_{n}-1+\xi\right)\right| d \xi,
$$

and using Lemma 2.2, we conclude that $G_{n} / 2$ and $\tilde{G}_{n} / 2$ are two complex monocomponents. It follows from $(2.10)$ and $F=2 \operatorname{Re}\left(F^{+}\right)$that

$$
\left\|F-\operatorname{Re}\left(e^{-i(\cdot)} \sum_{k=1}^{n}\left(G_{k}+\tilde{G}_{k}\right)\right)\right\|_{2} \leq 2\left\|\operatorname{Re}\left(F^{+}-\frac{e^{-i(\cdot)}}{2 \pi} \int_{1}^{N_{n}} e^{i \xi(\cdot)} \hat{F}_{1}(\xi) d \xi\right)\right\|_{2} \leq \epsilon_{n} .
$$

The proof is complete.

The corollaries of Theorem 1.3 may be proved similarly as for those of Corollary 1.1 and Corollary 1.2.

Remark 2.3. We note that in Theorem 1.3 and its corollaries the obtained decompositions are not quite into mono-components but with a multiplier $e^{-i s}$. After the phase modulation by $e^{i s}$ the results become decompositions into mono-components. If $0 \notin \operatorname{supp} \hat{F}$, then we decompose $F$ into a sum of mono-components.

Remark 2.4. In Theorem 1.3 and its corollaries we start from a function in $L^{2}(\mathbb{R})$ but end up with mono-components not in $L^{2}(\mathbb{R})$, but in a larger space $L^{2}\left(\mathbb{R}, \frac{d s}{1+s^{2}}\right)$. That is associated with the technique that we use. Precisely, that is because of the term $e^{i z}$ that we introduce to form the mono-components $G_{n}(z)$ and $\tilde{G}_{n}(z)$.

Remark 2.5. In the EMD decomposition (see [8]) one obtains a sequence of IMF signals $f_{1}, \ldots, f_{n}$, and the algorithm process ceases with a previously set threshold. As result of the algorithm, the first IMF, viz., $f_{1}$, is of the highest frequency, considered to be combined with the noises, and the frequencies of the following IMFs are consecutively decreasing. In the above decomposition based on Fourier expansion, the frequencies of the obtained mono-components are consecutively increasing, and the error term is a combination with the higher order frequencies and the noises. In engineering applications of EMD one throws away the first IMF and the residue behind the $n$-th IMF. In our decomposition the obtained mono-components, $T_{n}$ and $\tilde{T}_{n}$, are $p$-starlike functions. The real part of a $p$-starlike function has approximately $p$ maximal and $p$ minimal values. In the case of Theorem 1.1, for instance, each of $T_{n}$ or $\tilde{T}_{n}$ is the real part of some function in $S\left(p, N_{n}\right)$ with $p \geq N_{n}$ (see the proof of the theorem). The proof of Theorem 1.3 shows that the obtained mono-components 
$G_{n}$ and $\tilde{G}_{n}$ are of frequencies at least as high as $e^{i N_{n} z}$. We note that if set

$$
g_{n}\left(e^{i t}\right)=\operatorname{Re} \sum_{N_{n}+1 \leq|k| \leq N_{n+1}} c_{k} e^{i k t}
$$

or

$$
g_{n}(s)=\operatorname{Re} \int_{N_{n}<|\xi|<N_{n+1}} e^{i \xi s} \hat{F}_{1}(\xi) d \xi
$$

then the $g_{n}$ 's are of higher and higher frequencies, orthogonal to each other, and all of zero integral means, and they look like IMFs.

\section{Examples}

Below we provide two examples. The first one is for the compact interval case dealt with through Theorem 1.1. It is compared with an EMD decomposition. The second is for the infinite interval case dealt with by Corollary 1.5.

Example 3.1. Let function

$$
f(t)=\frac{\cos [2(t-\pi)]^{2}+5 \sin [2(t-\pi)]}{1+[2(t-\pi)]^{2}}
$$

be defined in $[-\pi, \pi]$.
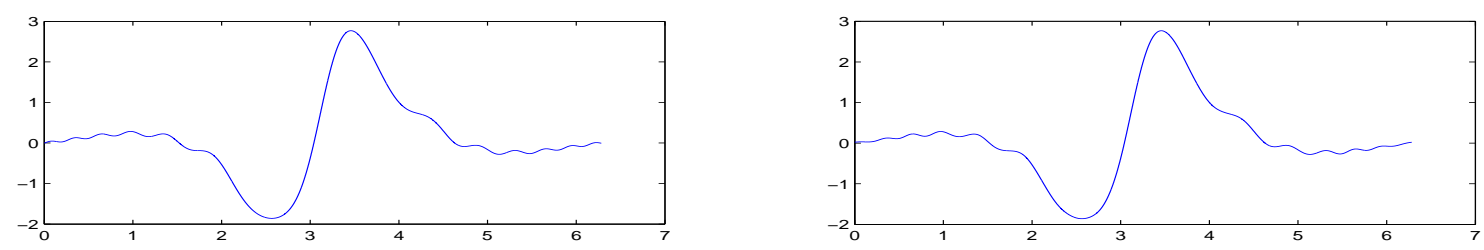

Figure 1. Left: Original function $f(t)$. Right: Fourier series $S_{25}(t)$ of $f(t)$ up to the 25th order, $\left\|f(t)-S_{25}(t)\right\|_{2}=9.1524 \times 10^{-5}$. 

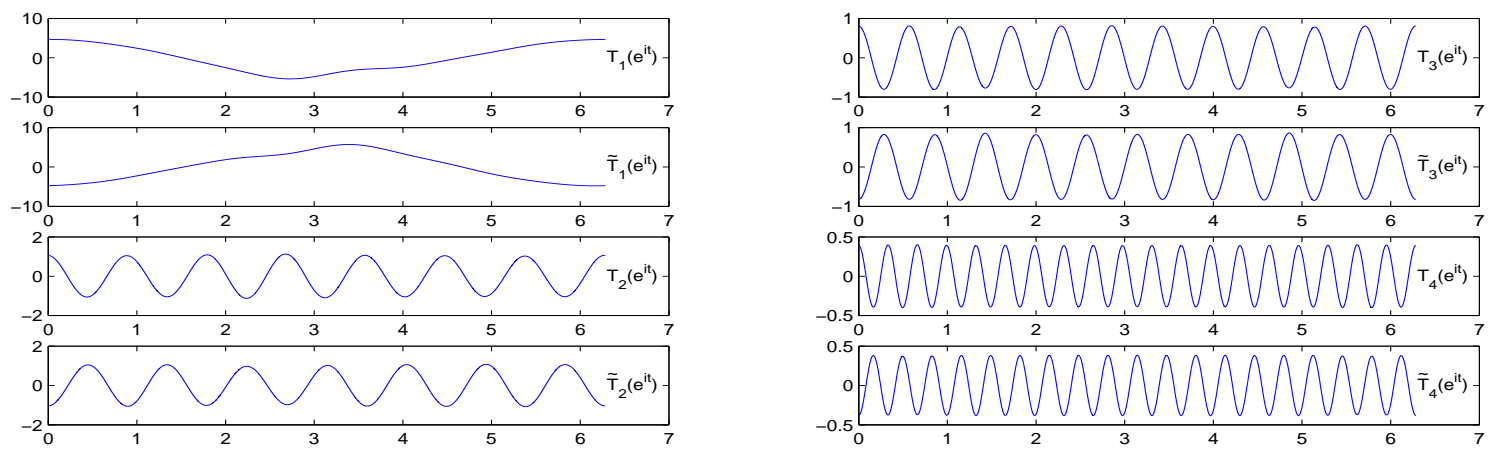

Figure 2. Decomposition of $S_{25}(t)$ into a sum of four real mono-components, where $\varepsilon_{1}=10^{-1}, \varepsilon_{2}=10^{-2}$, $\varepsilon_{3}=10^{-3}, \varepsilon_{4}=10^{-4}$, and $N_{1}=6, N_{2}=10, N_{3}=18, N_{4}=25$.

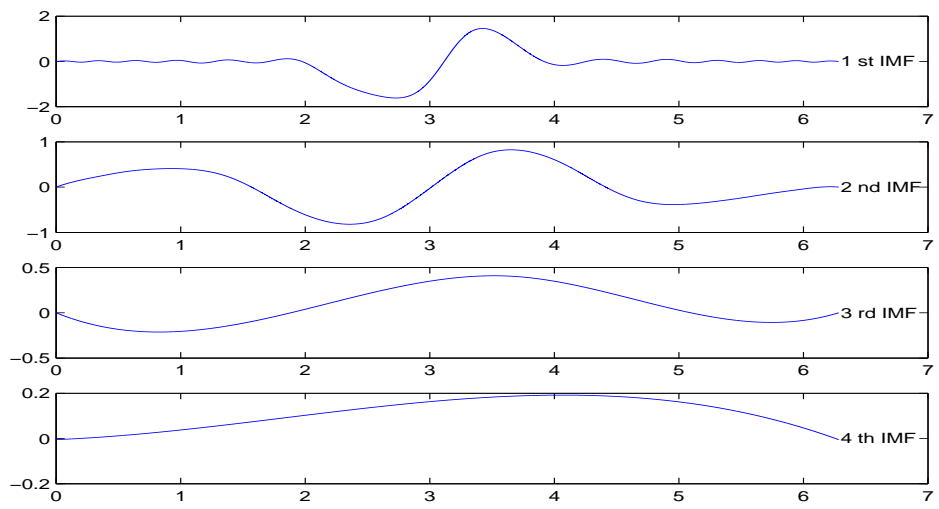

Figure 3. An EMD decomposition of the original function $f(t)$ into a sum of four IMFs, run by Matlab 7.3

Example 3.2. Consider function

$$
F(s)=\frac{1}{1+s^{2}}-0.7 e^{\frac{-s^{2}}{2}}
$$

defined on $\mathbb{R}$. Its Fourier transform

$$
\widehat{F}(\xi)=\pi e^{-|\xi|}-0.7 \times \sqrt{2 \pi} e^{-\frac{\xi^{2}}{2}},
$$

and its Hilbert transform

$$
H F(\xi)=\frac{\xi}{1+\xi^{2}}-0.7 \times \sqrt{\frac{2}{\pi}} \int_{0}^{\infty} e^{-\frac{x^{2}}{2}} \sin (x \xi) d x .
$$



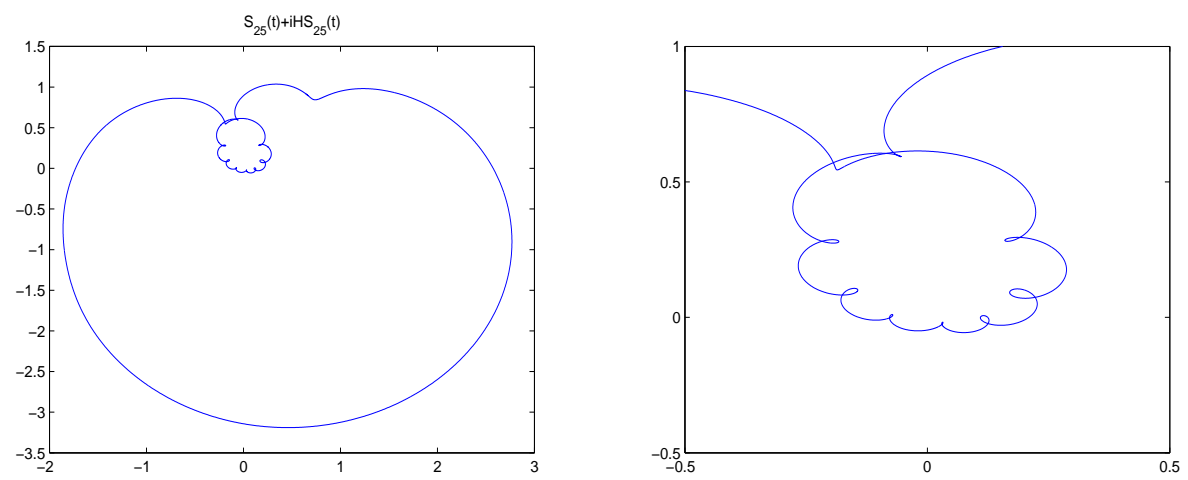

Figure 4. The figure on the right is enlargement of part of the figure on the left.
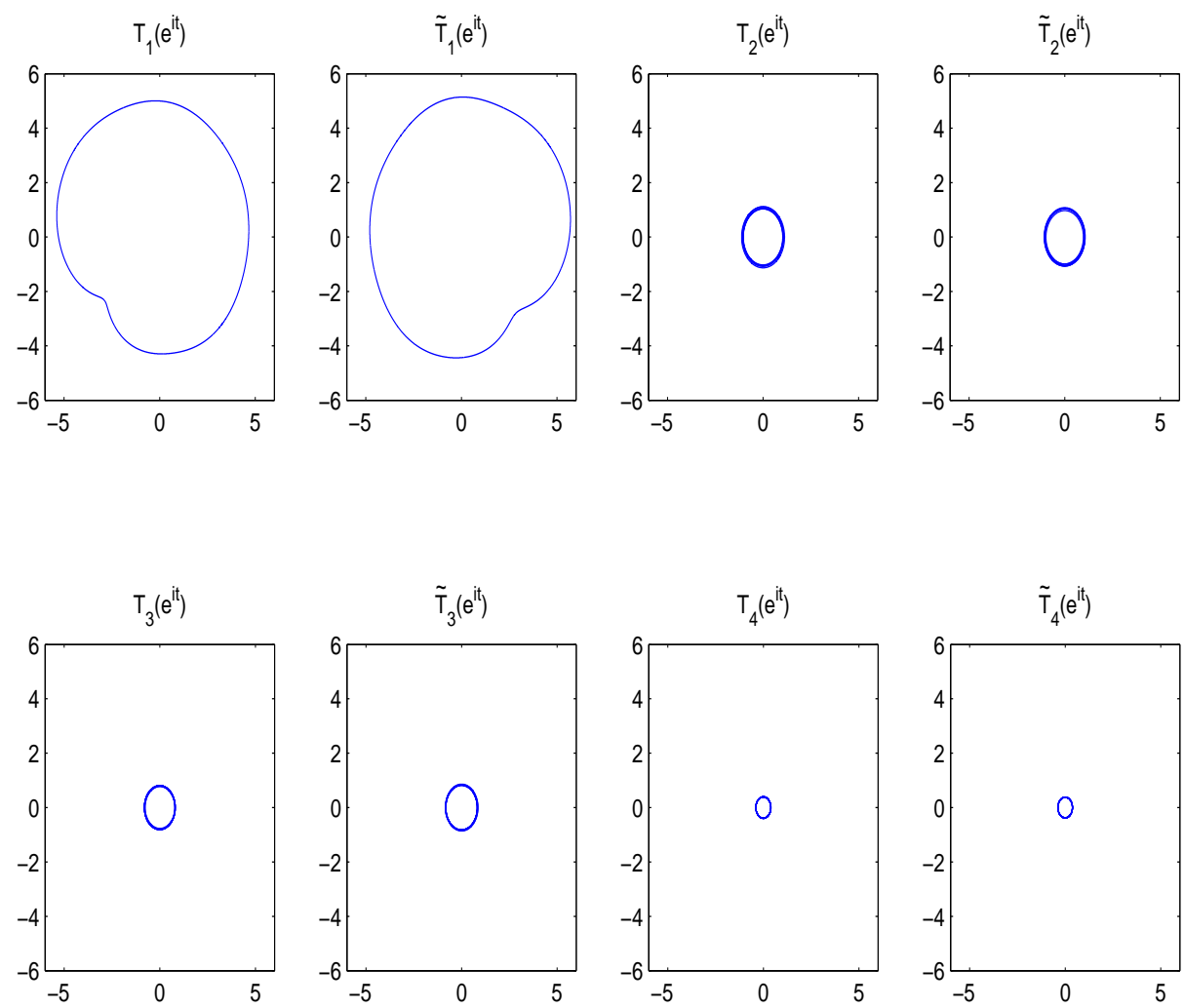

Figure 5. Analytic signals associated with the real signals $T_{1}\left(e^{i t}\right), \widetilde{T}_{1}\left(e^{i t}\right), T_{2}\left(e^{i t}\right), \widetilde{T}_{2}\left(e^{i t}\right), T_{3}\left(e^{i t}\right), \widetilde{T}_{3}\left(e^{i t}\right), T_{4}\left(e^{i t}\right)$ and $\tilde{T}_{4}\left(e^{i t}\right)$, respectively They appear like circles. If the scale is made large, then one can see perturbations on the circles of high winding numbers. 

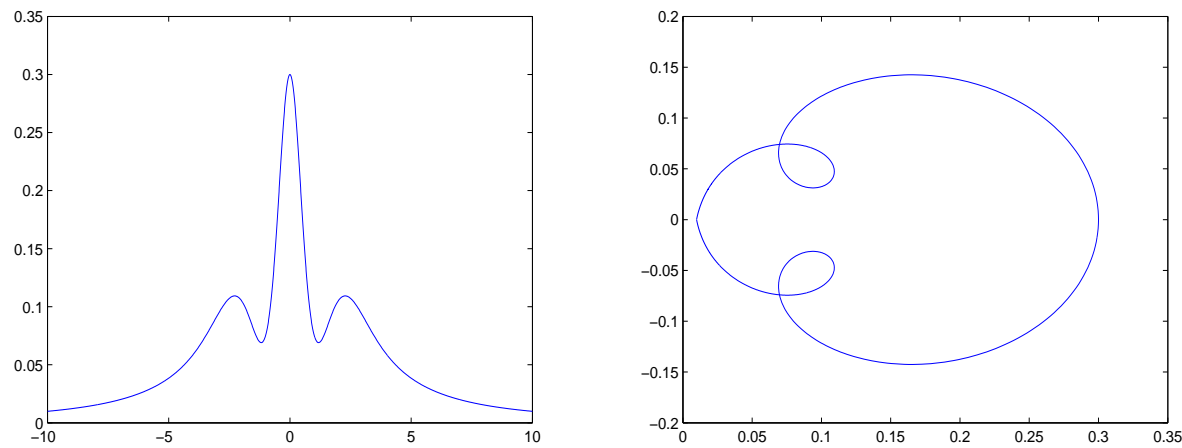

Figure 6. Left: The original function $f(t)$. Right: The associated analytic signal with $f(t)$, i.e. $f(t)+i H f(t)$ that is a multi-component.

It is clear that $F(s)$ is not a real mono-component, but satisfies the requirements of Corollary (1.11). Accordingly, we have the decomposition $F(s)=\operatorname{Re}\left[e^{-i s}\left(G_{1}(s)+\right.\right.$ $\left.\tilde{G}_{1}(s)\right]$, where $G_{1}, \widetilde{G}_{1}$, are complex mono-components.

$$
\begin{gathered}
G_{1}(s)=\frac{A e^{i s}}{2 \pi}+\frac{1}{2 \pi} \int_{1}^{\infty} e^{i s \xi} \widehat{F}(\xi-1) d \xi \\
\tilde{G}_{1}(s)=-\frac{A e^{i s}}{2 \pi}+\frac{1}{2 \pi} \int_{1}^{\infty} e^{i s \xi} \widehat{F}(\xi-1) d \xi .
\end{gathered}
$$

Since $\widehat{F}(\xi) \geqslant 0$, the number $A$ in the proof of Theorem 1.3 can be taken to be

$$
A=3>\int_{1}^{\infty} \xi \widehat{F}(\xi-1) d \xi=\int_{1}^{\infty} \xi\left(\pi e^{-\xi+1}-0.7 \sqrt{2 \pi} e^{-\frac{(\xi-1)^{2}}{2}}\right) d \xi \approx 2.3294 .
$$
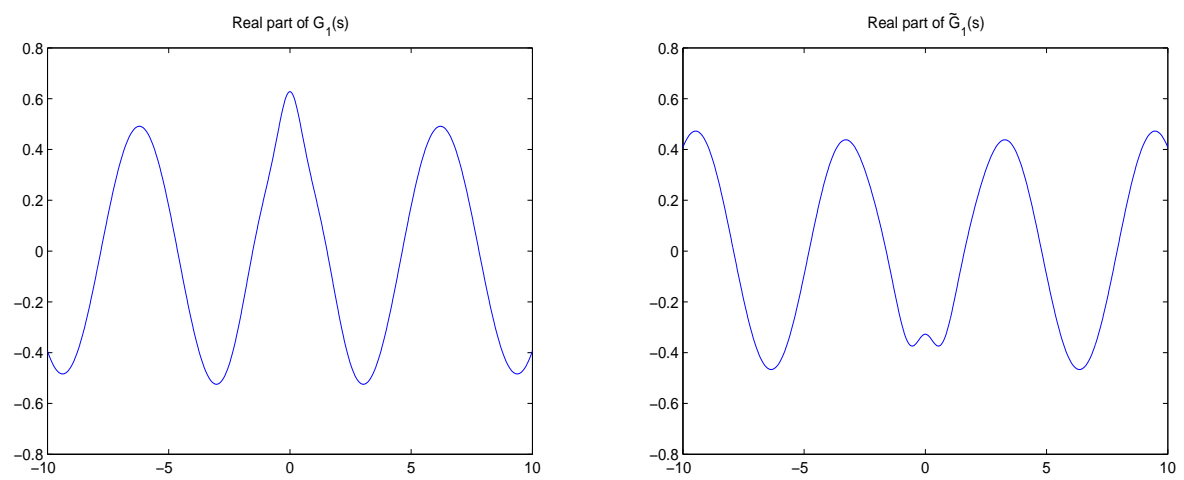

Figure 7. Real parts of $G_{1}$ and $\tilde{G}_{1}$ for $s$ between -10 and 10 

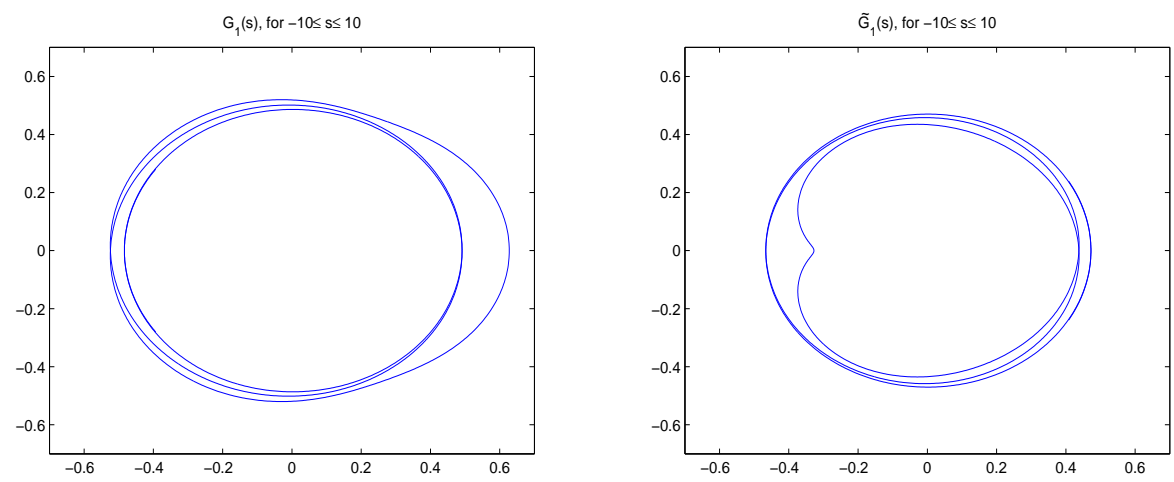

Figure 8. The analytic signals $G_{1}$ and $\tilde{G}_{1}$ for $s$ between -10 and 10 . They are mono-components.

Finally, we give the figure with the real part of $e^{-i s}\left(G_{1}(s)+\widetilde{G}_{1}(s)\right)$, where $\max _{-10<s_{k} \leq 10} \mid F\left(s_{k}\right)-\operatorname{Re}\left[e^{-i s_{k}}\left(G_{1}\left(s_{k}\right)+\widetilde{G}_{1}\left(s_{k}\right)\right] \mid \approx 2.2082 \times 10^{-4}\right.$, where $s_{k}=$ $-10+0.01 k, k=0,1,2, \ldots, 2000$.

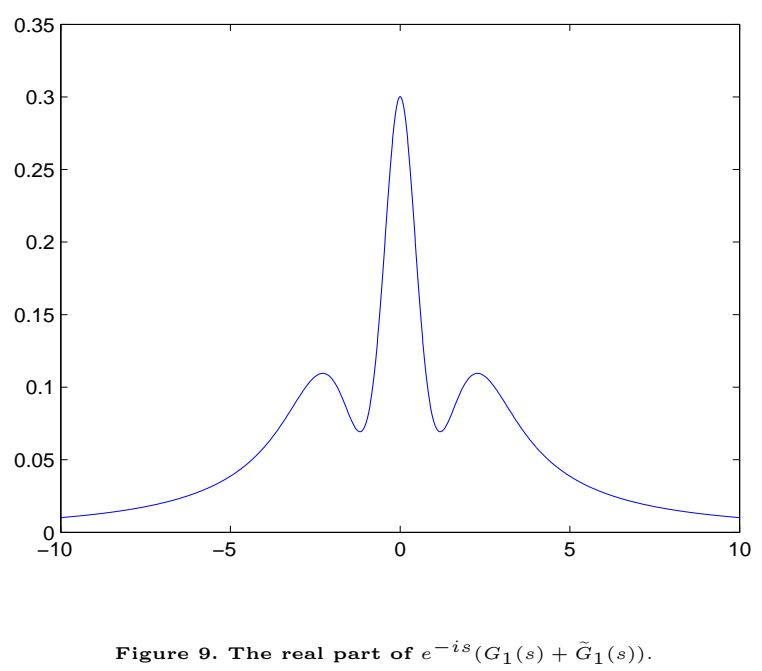

\section{Conclusion and Further Expectation}

Through the results proved in the previous section we show that theoretical and practical adaptive decomposition of signals into a small number of monocomponents is available. The mono-components in our decompositions are adaptive as they depend on sections of the Fourier series or those of the Fourier integral of 
the given signal. The convergence may be made as fast as one can possibly wish. From the current study there would be some aspects to be improved.

First, the composing mono-components obtained in our decompositions are of partial orthogonality but not total. Indeed, for instance in Theorem 1.4, any function in the pair $T_{k}$ and $\tilde{T}_{k}$ will be orthogonal with any function in the pair $T_{l}$ and $\tilde{T}_{l}$, where $k \neq l$. In particular, all $T_{k}$ and $\tilde{T}_{k}$ are orthogonal with $T_{0}$, that implies that the system have the integral-zero-mean property. On the other hand, however, $T_{k}$ and $\tilde{T}_{k}$ are not orthogonal with each other for any $k>0$. One would seek for adaptive decompositions into mono-components of the total orthogonality. The reason why we cannot make our system to be of total orthogonality is that the constants $A_{n}$ that we choose to construct the composing mono-components are very large and thus they prevent the obtained mono-components from being orthogonal to each other. Using a result in [22] may make the constants smaller but still large enough to prevent the constructed mono-components from being orthogonal to each other in the respective pairs.

Secondly, the ideal approach of adaptive decomposition into mono-components would be one similar to the EMD algorithm, but not based on Fourier expansions. What prevents us, at the present stage of the study, from having an algorithm like EMD, say, based on suitably adapted $M$ and $D$ operators or likewise, would be that the mono-component theory has intrinsic connection with conformal mappings. In the latter boundary interpolation does not directly imply approximation on the boundary. Algorithms like EMD but for mono-components would require deeper study in relation to conformal mappings.

\section{Acknowledgement}

The author would like to thank Prof Cheng, Qian-sheng, for helpful observations he provided in relation to spectrum decomposition of functions during his visit to University of Macau in 2006.

\section{References}

1. E. Bedrosian, A product theorem for Hilbert transform, Proc. IEEE 51(1963), 868-869.

2. B. Boashashe, Estimating and interpreting the instantaneous of a signal-Part 1: Fundamentals, Proc. IEEE, 80(1992), pp. 520-538, .

3. L. Cohn, Time-Frequency Analysis: Theory and Applications, 1995, Prentice Hall.

4. Q-H. Chen, L-Q. Li and T. Qian, Stability of frames generalized by non-linear Fourier atoms, International Journal of Waveletss, Multiresolution and Information Processing, Vol. 3, No.4 (2005), 1-12.

5. D. Gabor, Theory of Communications, Proc. IEE, vol. 93 (III), pp. 429-457.

6. J. B. Garnett, Bounded Analytic Functions, Academic Press, 1987.

7. A.W. Goodman and J.A. Hummel, A note on multivalent starlike functions, Proceedings of American Mathematical Society, Vol. 16,No.2, (1965), 284-288.

8. N. E. Huang et al, The empirical mode decomposition and the Hilbert spectrum for nonlinear and non-stationary time series analysis, Proc. R. Soc. Lndon, A454(1998), 903-995 
9. G. Jensen, Univalent Functions, 1975, Vandenhoeck \& Ruprecht.

10. B. Picinbono, On instantaneous amplitude and phase of signals, IEEE Transactions on Signal Processing, vol, 45, No. 3, (1997), 552-560.

11. T. Qian, Analytic signals and harmonic measures, J. Math. Anal. Appl., 314(2006) 526-536.

12. T. Qian, Characterization of Boundary Values of Functions in Hardy Spaces With Application in Signal Analysis, Journal of Integral Equations and Applications, Volume 13 Issue 2 (2005), 159-198.

13. T. Qian, Mono-components for decomposition of signals, Math. Meth. Appl. Sci., 29(2006), 1187-1198.

14. T. Qian, Phase Derivative of Nevanlinna Functions and Applications, accepted to appear in Math. Meth. Appl. Sci. (Online version: Early View at http://www3.interscience.wiley.com/journal/106560971/issue).

15. T. Qian, Q-H. Chen, and L-Q. Li, Analytic unit quadrature signals with nonlinear phase, Physica D, 203(2005), 80-87.

16. T. Qian, Y-S. Xu, D-Y. Yan, L-X. Yan and B. Yu, Fourier spectrum characterization of Hardy spaces and applications, accepted to appear in Proc. of A.M.S. (Available on the website of PAMS since September 11, 2008. Predicted to appear in the hard copy journal issue for March 2009).

17. T. Qian, L.-M. Zhang and H. Li, Mono-components vs IMFs in signal Decomposition, Mathematical Methods in the Applied Sciences, 29(2006), 1187-1198.

18. R.C. Sharpley and V. Vatchev, Analysis of intrinsic mode functions, Constr. Approx., 24(2006), 17-47.

19. W. Rudin, Real and complex analysis, McGraw-Hill International Editions 1987.

20. Y-S. Xu and D-Y. Yan, The Bedrosian identity for the Hilbert transform of product functions, Proc. of A. M. S., 134(2006), 2719-2728.

21. B. Yu and H-Z. Zhang, The Bedrosian Identity and Homogeneous Semi-Convolution Equations, accepted to appear in Journal of Integral Equations and Applications, Volume 20 Issue Number 4 (WINTER 2008).

22. D.-S. Zhou, Analytic sugnals of positive instantaneous frequencies, Master thesis, 2007, Peking Univrsity. 